\title{
PROTEINOGRAMA SÉRICO DE OVINOS INTOXICADOS EXPERIMENTALMENTE POR SALSA
}

\author{
Daniel Praseres Chaves ${ }^{1}$, José Jurandir Fagliari ${ }^{2}$, Paulo Cesar da Silva ${ }^{3}$ \\ ${ }^{1}$ Professor Doutor, Universidade Estadual do Maranhão - São Luiz, MA - daniel@cdvma.com.br \\ ${ }^{2}$ Professor Doutor, Faculdade de Ciências Agrárias e Veterinárias de Jaboticabal - UNESP \\ ${ }^{3}$ Auxiliar acadêmico do Laboratório de Pesquisa do Depto de Clínica e Cirurgia Veterinária - FCAV/UNESP - \\ Jaboticabal, SP
}

\begin{abstract}
Para avaliar a concentração sérica de proteínas de ovinos experimentalmente intoxicados por Ipomoea asarifolia, quatro grupos de cinco animais receberam dieta com inclusão de $25 \%$ (G1), 50\% (G2), 75\% (G3) e 100\% (G4) da planta em substituição ao Pennisetum purpureum. Amostras de sangue para proteinograma foram colhidas em sete momentos (M): antes do início do fornecimento de I. asarifolia (M1), cinco (M2), dez (M3), 15 (M4), 20 (M5), 25 (M6) e 30 (M7) dias após o início do tratamento. A concentração sérica de proteína total (PT) foi obtida por meio de espectrofotometria e as frações protéicas mediante eletroforese em gel de poliacrilamida (SDSPAGE). Detectou-se 29 a 31 proteínas no traçado
\end{abstract}

densitométrico, com pesos moleculares variando de 19.160 a 250.000 Dáltons (Da). Notou-se redução do teor de transferrina e aumento de $\alpha_{1}$-glicoproteína ácida em ovinos que ingeriram $75 \%$ e $100 \%$ da planta, imediatamente antes de morrerem. Também se constatou diminuição dos teores de IgG de cadeia pesada em M3 e M4 do G3, bem como de IgG de cadeia leve em M2, M3 e M4 desse mesmo grupo, sugerindo interferência na resposta imune dos ovinos intoxicados, especialmente naqueles com maior percentual de inclusão da planta na dieta. Concluiu-se que essas proteínas podem ser utilizadas como indicadores auxiliares no diagnóstico de intoxicação por I. asarifolia.

PALAVRAS-CHAVE: Ipomoea asarifolia; ovinos; plantas tóxicas.

\section{SERUM PROTEIN CONCENTRATION OF SHEEP EXPERIMENTALLY POISONED BY Ipomoea asarifolia}

\section{ABSTRACT}

In order to evaluate the serum protein concentration of sheep experimentally poisoned by Ipomoea asarifolia, four groups of five animals received diet with inclusion of $25 \%(\mathrm{G} 1), 50 \%(\mathrm{G} 2), 75 \%(\mathrm{G} 3)$ and $100 \%(\mathrm{G} 4)$ of the plant replacing to Pennisetum purpureum. Blood samples for serum protein concentration evaluation were collected at seven moments (M): before of the I. asarifolia supply (M1), and five (M2), ten (M3), 15 (M4), 20 (M5), 25 (M6) and 30 (M7) days after I. asarifolia supply. The serum concentration of total protein (TP) was measured through spectrophotometry and the protein fractions by means of eletrophoresis in poliacrylamide (SDS-PAGE). Twenty-nine to 31 proteins were detected by

KEYWORDS: Ipomoea asarifolia; poisonous plants; sheep. densitometric tracing, with molecular weights varying from 19,160 to 250,000 Dáltons (Da). Reduction of the transferrin concentration and increase of acid $\alpha_{1}$ glycoprotein level were verified in sheep that ingested $75 \%$ and $100 \%$ of the plant, immediately before dying. Also, reduction of concentration of IgG heavy chain was evident in M3 and M4, in G3 animals, as well as the level IgG light chain in M2, M3 and M4 of this group, suggesting interference in the immune function in poisoned sheep, especially in those with higher level of the plant on the diet. It is inferred that these proteins can be utilized as auxiliary indicators to diagnose intoxication by I. asarifolia. 


\section{INTRODUÇÃO}

Ipomoea asarifolia, popularmente conhecida como salsa, é uma planta tóxica pantropical, com ampla ocorrência no Brasil, principalmente nas regiões Norte e Nordeste (DÖBEREINER et al., 1960; AUSTIN \& CAVALCANTI, 1982). É abundante na região dos Lençóis Maranhenses, onde permanece verde durante todo $\mathrm{o}$ ano, atuando, em algumas áreas, como barreira natural ao deslocamento das dunas (CHAVES, 2009). É considerada causa da síndrome tremorgênica em ruminantes, doença caracterizada por alterações neurológicas, como desequilíbrio motor, tremores musculares, incoordenação, excitabilidade, queda e morte (TOKARNIA et al., 2002; GUEDES et al., 2007; ARAÚJO et al., 2008). É provável que a ingestão dessa planta seja responsável pela indução de perturbações na neurotransmissão pela interferência em mecanismos bioquímicos ainda não esclarecidos (BARBOSA et al., 2005).

As proteínas de fase aguda constituem um grupo de glicoproteínas sintetizadas principalmente no fígado, sendo sua produção estimulada por citocinas específicas, liberadas por leucócitos e macrófagos, com rápidas elevações de suas concentrações séricas em condições inflamatórias. Essas proteínas regulam a resposta inflamatória local e, na circulação sanguínea, induzem efeitos sistêmicos como febre e outras alterações metabólicas (DINARELLO, 1984; KENT, 1992), além de desempenharem diversas funções durante a fase inflamatória e a reparação tecidual. Assim, pode ser útil no diagnóstico, prognóstico e monitoramento terapêutico de doenças em animais domésticos. Essas proteínas são classificadas como positivas, representadas pela ceruloplasmina, fibrinogênio, proteína Creativa, antitripsina e haptoglobina, e como negativas - prealbumina, albumina e transferrina (KANEKO et al., 2008).

A imunidade adquirida por animais que ingerem sementes de Ricinus communis ou Abrus precatorius em doses crescentes, tornando-os resistentes à ingestão de quantidades múltiplas das doses letais (TOKARNIA et al., 2000), são exemplos que podem ser mencionados como indicadores de que algumas plantas tóxicas podem induzir reação imune. Segundo SOHNI \& BHATT (1996) e ZHANG (2004), diversas plantas podem ter atividades relacionadas ao sistema imune, estimulando-o ou suprimindo-o em níveis terapêuticos ou tóxicos.
No presente trabalho, objetivou-se avaliar o proteinograma de ovinos intoxicados experimentalmente com I. asarifolia, obtido em gel de poliacrilamida contendo dodecil sulfato de sódio (SDS-PAGE), visando propiciar subsídios laboratoriais ao diagnóstico e controle da intoxicação.

\section{MATERIAL E MÉTODOS}

Foram utilizados 20 ovinos deslanados clinicamente sadios sem padrão racial definido, machos e fêmeas, com seis a nove meses de idade e peso entre 17 e $23 \mathrm{~kg}$, obtidos de rebanhos particulares do município de Paulino Neves-MA, localizado na região dos Lençóis Maranhenses. O estudo foi aprovado pela Comissão de Ética e Bem-Estar Animal da FCAV/UNESP (parecer $n^{\circ}$ 013596-08).

Os ovinos foram distribuídos aleatoriamente em quatro grupos com cinco animais cada, os quais foram submetidos aos tratamentos apresentados no Quadro 1. Foi estabelecido um período de pré-experimento de oito dias, no qual os animais foram vermifugados com ivermectina (Ivomec ${ }^{\circledR}$ Solução Oral, Merial Saúde Animal, São Paulo-SP.), em dose oral recomendada pelo fabricante. Neste período, a alimentação consistiu de capim-elefante (Pennisetum purpureum) triturado, sal mineral e água à vontade. Caules, folhas e flores de I. asarifolia foram triturados e formulados como forrageira para fornecimento aos ovinos em diferentes níveis de substituição ao capim elefante.

Quadro 1. Percentual de inclusão de Ipomoea asarifolia e Pennisetum purpureum na dieta fornecida aos ovinos

\begin{tabular}{ccc}
\hline Grupo & $\begin{array}{c}\text { Ipomoea } \\
\text { asarifolia } \\
\%\end{array}$ & $\begin{array}{c}\text { Pennisetum } \\
\text { purpureum } \\
\%\end{array}$ \\
\hline 1 & 25 & 75 \\
2 & 50 & 50 \\
3 & 75 & 25 \\
4 & 100 & 0 \\
\hline
\end{tabular}

Amostras de sangue foram obtidas em sete momentos, com intervalos de cinco dias. As colheitas foram realizadas imediatamente antes do fornecimento de I. asarifolia (M1) e cinco (M2), dez (M3), 15 (M4), 20 (M5), 25 (M6) e 30 (M7) dias após o início do consumo do vegetal.

O teor de proteína total foi determinado pelo 
método do biureto e o de albumina pela técnica do bromocresol, utilizando-se conjunto de reagentes comerciais (Labtest, Labtest Diagnóstico S.A., Lagoa Santa, MG) e leitura em espectrofotômetro (Labquest mod. E-225-D, Labtest Diagnóstico S.A., Lagoa Santa, MG), em comprimento de onda específico. O proteinograma sérico foi obtido pela técnica de eletroforese em gel de poliacrilamida contendo dodecil sulfato de sódio (SDS-PAGE), conforme método descrito por LAEMMLI (1970). Após o fracionamento, o gel foi corado durante duas horas em solução de azul de coomassie; em seguida, foi descorado em solução de metanol e ácido acético. As concentrações das proteínas foram determinadas em densitômetro computarizado (Densitometer CS9301-Shimadzu, Tokyo-Japan). Como referência foi utilizada solução marcadora (Sigma Marker, Wide Range Saint Louis, USA) com diversos pesos moleculares, variando de 6.500 a 200.000 dáltons, bem como as proteínas purificadas $\operatorname{IgG}$, haptoglobina, $\alpha_{1}$-antitripsina, ceruloplasmina $\mathrm{e}$ transferrina.

Para análise estatística empregou-se o programa computacional Statistical Analysis System (SAS - versão 9.1) As comparações das médias foram feitas pelo teste Duncan a $5 \%$ de significância $(\mathrm{P} \leq 0,05)$.

\section{RESULTADOS E DISCUSSÃO}

Dos 20 ovinos, somente dois, pertencentes ao G1, permaneceram vivos até 30 dias após o início do experimento. Os sinais clínicos de intoxicação surgiram entre o $7^{\circ}$ e o $17^{\circ}$ dia após o início da ingestão de $25 \%$ da planta $(\mathrm{G} 1)$; entre o $4^{\circ}$ e o $8^{\circ}$ dia após ingestão de $50 \%(\mathrm{G} 2)$; entre o $2^{\circ}$ e o $5^{\circ}$ dia após ingestão de $75 \%(\mathrm{G} 3)$; e entre o $2^{\circ}$ e o $3^{\circ}$ dia após ingestão de $100 \%$ de salsa (G4). Ranger de dentes, hiperemia de membrana mucosa conjuntival, excitabilidade, apatia, tremor, desequilíbrio motor e queda foram os sinais clínicos observados nos ovinos intoxicados por $I$. asarifolia. A maioria dos sinais coincide com aqueles verificados em casos naturais da doença, descritos por DÖBEREINER et al. (1960), TOKARNIA et al. (2000), GUEDES et al. (2007) e ARAÚJO et al. (2008).

A concentração sérica de proteína total apresentou pequena oscilação, com menores valores em M7 do G1 (Tabela 1). O teor sérico de albumina foi menor em M3 a M6 do grupo 1 e no M4 do grupo 4 (Tabela 2).

Tabela 1. Médias e desvios-padrão da concentração sérica de proteína total $(\mathrm{g} / \mathrm{dL})$ de ovinos que receberam $25 \%(\mathrm{G} 1), 50 \%(\mathrm{G} 2), 75 \%(\mathrm{G} 3)$ e $100 \%(\mathrm{G} 4)$ de inclusão de Ipomoea asarifolia na dieta

\begin{tabular}{ccccc}
\hline Momento & $\mathrm{G} 1$ & $\mathrm{G} 2$ & $\mathrm{G} 3$ & $\mathrm{G} 4$ \\
\hline 1 & $8,02 \pm 0,95^{\mathrm{Aa}}$ & $7,88 \pm 0,86^{\mathrm{Aa}}$ & $7,90 \pm 1,06^{\mathrm{Aa}}$ & $7,27 \pm 0,45^{\mathrm{Aa}}$ \\
2 & $8,39 \pm 1,22^{\mathrm{Aa}}$ & $6,65 \pm 2,10^{\mathrm{Aa}}$ & $7,37 \pm 1,15^{\mathrm{Aa}}$ & $6,98 \pm 0,49^{\mathrm{Aa}}$ \\
3 & $7,37 \pm 0,64^{\mathrm{Aba}}$ & $6,69 \pm 1,12^{\mathrm{Aa}}$ & $7,50 \pm 0,76^{\mathrm{Aa}}$ & $6,36 \pm 0,13^{\mathrm{Aa}}$ \\
4 & $7,07 \pm 0,18^{\mathrm{Aba}}$ & $6,96 \pm 1,16^{\mathrm{Aa}}$ & $7,12 \pm 0,67^{\mathrm{Aa}}$ & $6,08 \pm 0,00^{\mathrm{Aa}}$ \\
5 & $6,33 \pm 0,29^{\mathrm{BCa}}$ & - & - & - \\
6 & $6,14 \pm 1,08^{\mathrm{BCa}}$ & - & - & - \\
7 & $5,57 \pm 0,29^{\mathrm{Ca}}$ & - & - & - \\
\hline
\end{tabular}

- Todos os animais do grupo morreram.

Valores seguidos de letras maiúsculas iguais na mesma coluna e minúsculas na mesma linha não diferem entre si pelo teste de Duncan $(\mathrm{p}>0,05)$.

No traçado densitométrico foram detectadas 29 a 31 proteínas, com pesos moleculares variando de 19.160 dáltons (Da) a 250.500 Da (Tabela 2). Dentre essas proteínas destacaram-se as de fase aguda, ceruloplasmina, $\quad \alpha 1$-antitripsina, transferrina, haptoglobina e glicoproteína ácida (GRUYS et al., 1994; FAGLIARI et al., 2007), bem como IgG, como as mais importantes no que diz respeito à intoxicação de ovinos pela salsa. Essas proteínas permitem avaliar a resposta imune dos animais e auxiliar no diagnóstico de diversos quadros clinicopatológicos (FAGLIARI \& SILVA, 2002; FAGLIARI et al., 2003; FAGLIARI et al., 2006; FERNANDÉZ et al., 2006). 
Tabela 2. Médias e desvios-padrão da concentração sérica das proteínas obtidas em gel de poliacrilamida (SDS-PAGE) de ovinos que receberam 25\% (G1), 50\% (G2), 75\% (G3) e 100\% (G4) de inclusão de Ipomoea asarifolia na dieta

\begin{tabular}{|c|c|c|c|c|c|c|c|}
\hline & M1 & M2 & M3 & M4 & M5 & M6 & M7 \\
\hline \multicolumn{8}{|c|}{ Imunoglobulina A } \\
\hline G1 & $20,3 \pm 2,4^{\mathrm{Bbc}}$ & $28,4 \pm 12,8^{\mathrm{BCbc}}$ & $18,9 \pm 11,4^{\mathrm{Bc}}$ & $25,4 \pm 20,3^{\mathrm{Abc}}$ & $28,4 \pm 2,4^{\mathrm{Abc}}$ & $99,2 \pm 46,3^{\mathrm{Aa}}$ & $53,3 \pm 27,6^{\mathrm{Ab}}$ \\
\hline $\mathrm{G} 2$ & $23,6 \pm 2,7^{\mathrm{Bb}}$ & $20,8 \pm 11,3^{\mathrm{Cb}}$ & $36,2 \pm 22,7^{\mathrm{ABab}}$ & $52,5 \pm 5,6^{\mathrm{Aa}}$ & - & - & - \\
\hline G3 & $34,2 \pm 13,2^{\mathrm{Aba}}$ & $19,7 \pm 11,7^{\mathrm{Ca}}$ & $29,3 \pm 13,2^{\mathrm{ABa}}$ & $31,5 \pm 0,4^{\mathrm{Aa}}$ & - & - & - \\
\hline G4 & $44,0 \pm 20,0^{\mathrm{ABab}}$ & $14,1 \pm 3,7^{\mathrm{Cb}}$ & $48,7 \pm 7,7^{\mathrm{ABa}}$ & $54,1 \pm 0,0^{\mathrm{Aa}}$ & - & - & - \\
\hline \multicolumn{8}{|c|}{ Ceruloplasmina } \\
\hline G1 & $28,3 \pm 4,4^{\mathrm{Bab}}$ & $32,4 \pm 13,9^{\mathrm{Aab}}$ & $19,2 \pm 7,3^{\mathrm{Ab}}$ & $21,9 \pm 8,8^{\mathrm{Ab}}$ & $21,4 \pm 2,6^{\mathrm{Ab}}$ & $51,7 \pm 34,4^{\mathrm{Aa}}$ & $32,0 \pm 17,6^{\mathrm{Aab}}$ \\
\hline $\mathrm{G} 2$ & $30,4 \pm 13,4^{\mathrm{Ba}}$ & $32,3 \pm 6,3^{\mathrm{Aa}}$ & $26,7 \pm 7,6^{\mathrm{Aab}}$ & $14,5 \pm 3,2^{\mathrm{Ab}}$ & - & - & - \\
\hline G3 & $54,4 \pm 21,4^{\mathrm{Aa}}$ & $30,6 \pm 12,2^{\mathrm{Aab}}$ & $23,2 \pm 3,6^{\mathrm{Ab}}$ & $29,5 \pm 12,7^{\mathrm{Aab}}$ & - & - & - \\
\hline G4 & $54,0 \pm 11,6^{\mathrm{Aa}}$ & $22,9 \pm 5,5^{\mathrm{Ab}}$ & $22,9 \pm 2,2^{\mathrm{Ab}}$ & $26,1 \pm 0,0^{\mathrm{Ab}}$ & - & - & - \\
\hline \multicolumn{8}{|c|}{ Transferrina } \\
\hline G1 & $321 \pm 208^{\mathrm{Aa}}$ & $304 \pm 139^{\mathrm{ABa}}$ & $422 \pm 134^{\mathrm{Aa}}$ & $437 \pm 109^{\mathrm{Aa}}$ & $358 \pm 78^{\mathrm{Aa}}$ & $351 \pm 117^{\mathrm{Aa}}$ & $327 \pm 41^{\mathrm{Aa}}$ \\
\hline G2 & $410 \pm 69^{\mathrm{Aa}}$ & $254 \pm 105^{\mathrm{Bb}}$ & $389 \pm 108^{\text {Aab }}$ & $459 \pm 69^{\mathrm{Aa}}$ & - & - & - \\
\hline G3 & $431 \pm 60^{\mathrm{Aa}}$ & $379 \pm 96^{\mathrm{ABa}}$ & $357 \pm 60^{\mathrm{Aa}}$ & $389 \pm 78^{\mathrm{Aa}}$ & - & - & - \\
\hline G4 & $449 \pm 169^{\mathrm{Aa}}$ & $458 \pm 177^{\mathrm{Aa}}$ & $397 \pm 66^{\mathrm{Aa}}$ & $183 \pm 0^{\mathrm{Ba}}$ & - & - & - \\
\hline \multicolumn{8}{|c|}{ Albumina } \\
\hline G1 & $4.432 \pm 476^{\mathrm{Aa}}$ & $4.565 \pm 3911^{\mathrm{Aa}}$ & $3.969 \pm 354^{\text {Aab }}$ & $3.725 \pm 96^{\mathrm{Ab}}$ & $3.120 \pm 307^{\mathrm{Ac}}$ & $2.846 \pm 608^{\text {Acd }}$ & $2.485 \pm 123^{\mathrm{Ad}}$ \\
\hline G2 & $4.132 \pm 669^{\mathrm{Aba}}$ & $3.468 \pm 988^{\mathrm{Ba}}$ & $3.487 \pm 560^{\mathrm{Aa}}$ & $3.584 \pm 644^{\mathrm{Aa}}$ & - & - & - \\
\hline G3 & $4.038 \pm 581^{\mathrm{Aba}}$ & $3.817 \pm 560^{\mathrm{ABa}}$ & $3.983 \pm 471^{\mathrm{Aa}}$ & $3.575 \pm 611^{\mathrm{Aa}}$ & - & - & - \\
\hline G4 & $3.773 \pm 185^{\mathrm{Aba}}$ & $3.755,3 \pm 152^{\mathrm{Ba}}$ & $3.751 \pm 339^{\mathrm{Aa}}$ & $3.794 \pm 0^{\mathrm{Aa}}$ & - & - & - \\
\hline \multicolumn{8}{|c|}{$\alpha_{1}$-antitripsina } \\
\hline G1 & $308 \pm 50^{\mathrm{Aa}}$ & $298 \pm 43^{\mathrm{Aa}}$ & $264 \pm 45^{\mathrm{Aa}}$ & $265 \pm 39^{\mathrm{Aa}}$ & $270 \pm 69^{\mathrm{Aa}}$ & $270 \pm 74^{\mathrm{Aa}}$ & $241 \pm 40^{\mathrm{Aa}}$ \\
\hline G2 & $307 \pm 35^{\mathrm{Aa}}$ & $238 \pm 41^{\mathrm{Ab}}$ & $237 \pm 46^{\mathrm{Ab}}$ & $308 \pm 50^{\mathrm{Aa}}$ & - & - & - \\
\hline G3 & $281 \pm 36^{\mathrm{Aa}}$ & $277 \pm 44^{\mathrm{Aa}}$ & $257 \pm 82^{\mathrm{Aa}}$ & $271 \pm 60^{\mathrm{Aa}}$ & - & - & - \\
\hline G4 & $281 \pm 58^{\mathrm{Aa}}$ & $291 \pm 37^{\mathrm{Aa}}$ & $240 \pm 14^{\mathrm{Aa}}$ & $260 \pm 0^{\mathrm{Aa}}$ & - & - & - \\
\hline \multicolumn{8}{|c|}{ Imunoglobulina G (cadeia pesada) } \\
\hline G1 & $1.370 \pm 321^{\mathrm{Aa}}$ & $1.577 \pm 492^{\mathrm{Aa}}$ & $1.325 \pm 232^{\mathrm{Aa}}$ & $1.341 \pm 172^{\mathrm{ABa}}$ & $1.289 \pm 153^{\mathrm{Aa}}$ & $1.349 \pm 292^{\mathrm{Aa}}$ & $1.302 \pm 270^{\mathrm{Aa}}$ \\
\hline G2 & $1.384 \pm 174^{\mathrm{Aa}}$ & $1.349 \pm 500^{\mathrm{Aa}}$ & $1.272 \pm 326^{\mathrm{ABa}}$ & $1.261 \pm 195^{\mathrm{Ba}}$ & - & - & - \\
\hline G3 & $1.553 \pm 286^{\mathrm{Aa}}$ & $1.413 \pm 275^{\mathrm{Aa}}$ & $1.462 \pm 127^{\mathrm{Aa}}$ & $1.616 \pm 22^{\mathrm{Aa}}$ & - & - & - \\
\hline G4 & $1.374 \pm 233^{\mathrm{Aa}}$ & $1.157 \pm 270^{\mathrm{Aa}}$ & $965 \pm 159^{\mathrm{Ba}}$ & $836 \pm 0$ Ca & - & - & - \\
\hline \multicolumn{8}{|c|}{ Imunoglobulina G (cadeia leve) } \\
\hline G1 & $846 \pm 263^{\mathrm{Aa}}$ & $963 \pm 324^{\mathrm{Aa}}$ & $797 \pm 227^{\mathrm{Aa}}$ & $747 \pm 108^{\mathrm{ABa}}$ & $731 \pm 70^{\mathrm{Aa}}$ & $632 \pm 165^{\mathrm{Aa}}$ & $642 \pm 61^{\mathrm{Aa}}$ \\
\hline G2 & $949 \pm 240^{\mathrm{Aa}}$ & $740 \pm 342^{\mathrm{Aa}}$ & $713 \pm 207^{\mathrm{Aa}}$ & $630 \pm 113^{\mathrm{Ba}}$ & - & - & - \\
\hline G3 & $882 \pm 247^{\mathrm{Aa}}$ & $829 \pm 236^{\mathrm{Aa}}$ & $881 \pm 106^{\mathrm{Aa}}$ & $907 \pm 132^{\mathrm{Aa}}$ & - & - & - \\
\hline G4 & $740 \pm 161^{\mathrm{Aa}}$ & $661 \pm 208^{\mathrm{Aa}}$ & $443 \pm 124^{\mathrm{Ba}}$ & $401 \pm 0^{\mathrm{Ca}}$ & - & - & - \\
\hline
\end{tabular}


continuação.

\begin{tabular}{|c|c|c|c|c|c|c|c|}
\hline & M1 & M2 & M3 & M4 & M5 & M6 & M7 \\
\hline \multicolumn{8}{|c|}{ Haptoglobina } \\
\hline G1 & $20,2 \pm 5,2^{\mathrm{Aa}}$ & $27,6 \pm 4,5^{\mathrm{Aa}}$ & $22,4 \pm 8,3^{\mathrm{Aa}}$ & $22,2 \pm 6,8^{\mathrm{Aa}}$ & $19,4 \pm 10,9^{\mathrm{Aa}}$ & $18,9 \pm 2,8^{\mathrm{Aa}}$ & $26,8 \pm 5,0^{\mathrm{Aa}}$ \\
\hline G2 & $20,5 \pm 11,2^{\mathrm{Aa}}$ & $23,8 \pm 9,4^{\mathrm{Aa}}$ & $22,3 \pm 8,3^{\mathrm{Aa}}$ & $18,7 \pm 6,5^{\mathrm{Aa}}$ & - & - & - \\
\hline G3 & $17,9 \pm 12,3^{\mathrm{Aa}}$ & $29,9 \pm 6,9^{\mathrm{Aa}}$ & $20,7 \pm 7,1^{\mathrm{Aa}}$ & $21,4 \pm 4,0^{\mathrm{Aa}}$ & - & - & - \\
\hline G4 & $24,8 \pm 8,8^{\mathrm{Aa}}$ & $31,6 \pm 9,5^{\mathrm{Aa}}$ & $22,2 \pm 1,3^{\mathrm{Aa}}$ & $13,9 \pm 0,0^{\mathrm{Aa}}$ & - & - & - \\
\hline \multicolumn{8}{|c|}{$\alpha_{1}$-glicoproteína ácida } \\
\hline G1 & $52,9 \pm 12,4^{\mathrm{Aa}}$ & $54,1 \pm 17,4^{\mathrm{Aa}}$ & $45,8 \pm 19,4^{\mathrm{Aa}}$ & $36,4 \pm 18,2^{\mathrm{BCa}}$ & $44,5 \pm 12,9^{\mathrm{Aa}}$ & $49,5 \pm 41,5^{\mathrm{Aa}}$ & $52,9 \pm 24,8^{\mathrm{Aa}}$ \\
\hline G2 & $57,1 \pm 31,9^{\mathrm{Aab}}$ & $34,0 \pm 17,9^{\mathrm{Bb}}$ & $39,1 \pm 17,2^{\mathrm{Ab}}$ & $74,5 \pm 2,9^{\mathrm{Aa}}$ & - & - & - \\
\hline G3 & $45,8 \pm 18,1^{\mathrm{Aa}}$ & $51,1 \pm 8,5^{\mathrm{ABa}}$ & $35,6 \pm 14,8^{\mathrm{Aa}}$ & $28,8 \pm 8,7^{\mathrm{Ca}}$ & - & - & - \\
\hline G4 & $45,1 \pm 8,7^{\mathrm{Ab}}$ & $49,2 \pm 7,8^{\mathrm{ABb}}$ & $23,3 \pm 7,2^{\mathrm{Ac}}$ & $75,4 \pm 0,0^{\mathrm{Aa}}$ & - & - & - \\
\hline \multicolumn{8}{|c|}{ Não identificada } \\
\hline G1 & $234 \pm 71^{\mathrm{Aab}}$ & $249 \pm 49^{\mathrm{Aa}}$ & $216 \pm 36^{\mathrm{ABabc}}$ & $213 \pm 28^{\mathrm{ABabc}}$ & $163 \pm 23^{\mathrm{Abc}}$ & $149 \pm 27^{\mathrm{Ac}}$ & $153 \pm 27^{\mathrm{Ac}}$ \\
\hline $\mathrm{G} 2$ & $264 \pm 24^{\mathrm{Aa}}$ & $211 \pm 69^{\mathrm{Aa}}$ & $207 \pm 39^{\mathrm{ABa}}$ & $214 \pm 41^{\mathrm{ABa}}$ & - & - & - \\
\hline G3 & $253 \pm 77^{\mathrm{Aa}}$ & $238 \pm 57^{\mathrm{Aa}}$ & $234 \pm 19^{\mathrm{ABa}}$ & $163 \pm 43^{\mathrm{BCa}}$ & - & - & - \\
\hline G4 & $222 \pm 39^{\mathrm{Aa}}$ & $225 \pm 21^{\mathrm{Aa}}$ & $189 \pm 1^{\mathrm{Bb}}$ & $148 \pm 0^{\mathrm{Cb}}$ & - & - & - \\
\hline
\end{tabular}

- Todos os animais do grupo morreram.

Valores seguidos de letras maiúsculas iguais na mesma coluna e minúsculas na mesma linha não diferem entre si pelo teste de Duncan ( $\mathrm{p}>0,05)$.

A despeito de diferenças observadas entre os grupos e os momentos quanto às concentrações séricas de Ig A, ceruloplasmina, transferrina, albumina, $\operatorname{IgG}$ de cadeia pesada, haptoglobina, $\alpha_{1^{-}}$ glicoproteína ácida, $\operatorname{IgG}$ de cadeia leve e da proteína de peso molecular 24.000 Da, pode-se inferir que os ovinos intoxicados por I. asarifolia não apresentaram sintomas indicativos de reação inflamatória sistêmica. Essa afirmação se baseia no fato de que as proteínas ceruloplasmina, haptoglobina, $\alpha_{1^{-}}$ antitripsina e $\alpha_{1}$-glicoproteína ácida que são proteínas de fase aguda (GRUYS et al., 1994; FAGLIARI et al., 2007), cujos teores séricos se elevam ainda na fase inicial da resposta inflamatória (THRALL et al., 2007), tiveram seus níveis séricos oscilando durante todo o período experimental.

A diminuição na concentração sérica de transferrina, constatada no M4 do grupo 4 indica um processo inflamatório isolado e essa redução é considerada normal, uma vez que a proteína é considerada de fase aguda negativa (TAKIGUCHI et al., 1990; TRUMEL et al., 1996; KANEKO et al., 1997). A concentração sérica de $\alpha_{1}$-glicoproteína ácida diminuiu nos ovinos dos grupos G2 e G4 até M3 e, em seguida, apresentou brusca elevação no M4 desses dois grupos. Essa elevação repentina é um fato considerado como uma característica das proteínas de fase aguda, segundo KANEKO et al. (2008). No estudo ficou evidente a redução do teor de transferrina e o aumento da concentração de $\alpha_{1}$ glicoproteína ácida em ovinos que ingeriram $75 \%$ e $100 \%$ da planta, imediatamente antes de morrerem.

Quanto aos teores séricos de $\operatorname{IgG}$ de cadeia pesada, notou-se diminuição em M3 e M4 nos animais do grupo 4, bem como de IgG de cadeia leve em M2, M3 e M4 nos ovinos desse mesmo grupo, sugerindo uma interferência na resposta imune dos ovinos intoxicados, especialmente naqueles com maior nível de inclusão de salsa na dieta.

A concentração sérica da proteína com 24.000 Da aumentou no M2 do grupo 1 e diminuiu no M4 dos grupos 2 e 3, bem como nos M3 e M4 do grupo 4, indicando que o percentual de inclusão de $I$. asarifolia na dieta dos ovinos influenciou a dinâmica dessa proteína ao longo do experimento. $\mathrm{Na}$ literatura não há relato de constatação dessa proteína em ovinos, tampouco de sua função. Os resultados indicam que parece se tratar de uma proteína de fase aguda negativa. 


\section{CONCLUSÕES}

O modelo experimental permitiu reafirmar a toxicidade da $I$. asarifolia em razão das elevadas taxas de morbidade e de mortalidade verificada nos ovinos alimentados com a planta. Ademais, esta pesquisa caracterizou a cinética das proteínas séricas de ovinos intoxicados por I. asarifolia, propiciando subsídios laboratoriais auxiliares úteis no diagnóstico e progressão da intoxicação, destacando-se a $\alpha_{1-}$ glicoproteína ácida e a transferrina. A primeira apresenta maior valor diagnóstico devido à precocidade, pois sua concentração sérica diminui no início e só eleva-se nos momentos terminais, quando o prognóstico pode ser considerado desfavorável.

\section{REFERÊNCIAS}

ARAÚJO, J.A.S.; RIET-CORREA, F.; MEDEIROS, R.M.T.; SOARES, M.P.; OLIVEIRA, D.M.; CARVALHO, F.K.L. Intoxicação experimental por Ipomoea asarifolia (Convolvulaceae) em caprinos e ovinos. Pesquisa Veterinária Brasileira, v.28 n.10, p.488-494, 2008.

AUSTIN, D.F.; CAVALCANTI, P.B. Convolvuláceas da Amazônia. Publicações Avulsas do Museu Goeldi, v.36, p.1-134, 1982.

BARBOSA, J.D.; OLIVEIRA, C.M.C. de; DUARTE, M.D.; PEIXOTO, P.V.; TOKARNIA,C.H. Intoxicação experimental e natural por Ipomoea asarifolia (Convolvulaceae) em búfalos e outros ruminantes. Rio de Jarneiro: Pesquisa Veterinária Brasileira, v.25, n.4, p.231-234, 2005.

BORGES, A.S.; FEITOSA, F.L.F.; BENESI, F.J.; BIRGEL E.H.; MENDES, L.C.N. Influência da forma de administração e da quantidade fornecida de colostro sobre a concentração de proteína total e de suas frações eletroforéticas no soro sangüíneo de bezerros da raça Holandesa. Arquivos Brasileiros de Medicina Veterinária e Zootecnia, v.53, n.5, p.629-634, 2001.

CHAVES, D.P. Intoxicação experimental por Ipomoea asarifolia em ovinos: achados clínicos, laboratoriais e anatomopatológicos. 2009. 82f. Tese (Doutorado em Medicina Veterinária) - Universidade Estadual Paulista, Jaboticabal.

(http://www.fcav.unesp.br/download/pgtrabs/cmv/d/3571)

DÖBEREINER, J.; TOKARNIA, C. H.; CANELA, C. F. C. Intoxicação experimental pela salsa Ipomoea asarifolia (R. et Schult) em ruminantes. Arquivos do Instituto Biológico Animal, v.3, p.39-57, 1960.

FAGLIARI, J.J.; PASSIPIERI, M.; OKUDA, H.T.; SILVA, S.L.; SILVA, P.C. 2007. Serum protein concentrations, including acute phase proteins, in calves with hepatogenous photosensitization. Arquivos Brasileiros de Medicina Veterinária e Zootecnia, v.59, n.6, p.1355-1358, 2007.

FAGLIARI, J.J.; RIZOLLI, F.W.; SILVA, S.L.; SILVA, D.G. Proteinograma sérico de bezerros recém-nascidos da raça Holandesa obtido por eletroforese em gel de poliacrilamida. Arquivos Brasileiros de Medicina Veterinária e Zootecnia, v.58, n.3, p.450-453, 2006.

FAGLIARI, J.J.; SILVA, S.L. Hemograma e proteinograma plasmático de eqüinos hígidos e de eqüinos acometidos por abdome agudo, antes e após laparotomia. Arquivos Brasileiros de Medicina Veterinária e Zootecnia, v.54, p.559-567, 2002.

FAGLIARI, J.J.; WEISS, D.J.; McCLENAHAN, D.; EVANSON, O.A. Serum protein concentrations in calves with experimentally induced pneumonic pasteurellosis. Arquivos Brasileiros de Medicina Veterinária e Zootecnia, v.55, p.383-387, 2003.

FERNANDÉZ, S.Y.; JESUS, E.E.V.; PAULE, B.J.A.; UZÊDA, R.S.; ALMEIDA, M.A.O.; GUIMARÃES, J.E. Proteinograma de caprinos da raça Pardo-Alpina infectados naturalmente por parasitos gastrintestinais. Arquivos Brasileiros de Medicina Veterinária e Zootecnia, v.58, n.2, p.279-282, 2006.

GRUYS, E.; OBWOLO, M.J.; TOUSSAINT M.J.M. Diagnostic significance of the major acute phase proteins in veterinary clinical chemistry: a review. Veterinary Bulletin, v.64, p.1009-1018, 1994.

GUEDES, K.M.R.; RIET-CORREA, F.; DANTAS, A.F.M.; SIMÕES, F.V.D.; MIRANDA-NETO, E.G.; NOBRE, V.M.T.; MEDEIROS, R.M.T. Doenças do sistema nervoso central em caprinos e ovinos no semiárido. Pesquisa Veterinária Brasileira., v.27, n.1, p.29$38,2007$.

KANEKO, J. J.; HARVEY, J. W.; BRUSS, M. L. Clinical Biochemistry of Domestic Animals. 5 ed. San Diego: Academic Press, 2008. 916p.

LAEMMLI, U.K. Cleavage of structural proteins during the assembly of the head of bacteriophage T4. Nature, v.277, p.680-685, 1970.

SOHNI, Y.R.; BHATT, R.M. Activity of a crude extract formulation in experimental hepatic amoebiasis and in immunomodulation studies. Journal of Ethnopharmacology, v.534 n.2-3, p.119-24, 1996.

TAKIGUCHI, M.; FUJINAGA, T.; NAIKI, M.; MISUNO, S.; OTOMO, K. Isolation, characterization, and quantitative analysis of c-reative protein from horses. American Journal Veterinary Research, v.51, p.12151220, 1990.

THRALL, M.A.; BACKER, D.C.; CAMPBELL, T.W.; DENICOLA, D.; FETTMAN, M.J.; LASSEN, E.D.; REBAR, A.; WEISER, G. Hematologia e bioquímica clínica veterinária. São Paulo: Roca, 2007. 582p.

TOKARNIA, C. H.; DÖBEREINER, J.; PEIXOTO, P. V. Plantas Tóxicas do Brasil. Rio de Janeiro: Helianthus, 2000. 320p. 
TOKARNIA, C.R.; DÖBEREINER, J.; PEIXOTO, P.V. $\quad$ 130, 1996.

Poisonous plants affecting livestock in Brazil. Toxicon, v.40, p.1635-1660, 2002.

ZHANG, L; WANG, Y; WANG, L.Z.; GAO, X.M. Immunopotentiating effect of a yang.- promoting formula

TRUMEL, C.; SCHELCHER, F.; BRAUM, J. P.; of traditional chinese medicine on aged female Balb/c GUELFI, J. F. L’lectrophorese des protéines sériques: mice. Phytotherapy Research, v.18, n.10, p.857-861, principes d' interpretation chez le chien, le chat et le 2004.

cheval. Revue de Medecine Veterinaire, v.147, p.123-

Protocolado em: 10 maio 2010. Aceito em: 15 jun. 2011. 